Service social

\title{
Une intervention sur le sida dans une école secondaire
}

\section{Vivianne Vachon et Marie Berlinguet}

Volume 38, numéro 1, 1989

Aspects psychosociaux du Sida

URI : https://id.erudit.org/iderudit/706425ar

DOI : https://doi.org/10.7202/706425ar

Aller au sommaire du numéro

\section{Éditeur(s)}

École de service social de l'Université Laval

\section{ISSN}

1708-1734 (numérique)

Découvrir la revue

\section{Citer cet article}

Vachon, V. \& Berlinguet, M. (1989). Une intervention sur le sida dans une école secondaire. Service social, 38(1), 78-84. https://doi.org/10.7202/706425ar

\section{Résumé de l'article}

Cet article décrit une brève intervention éducative et préventive sur le sida effectuée dans une école secondaire de la Commission scolaire des Découvreurs, dans la région de Québec. La collaboration du personnel de l'école et du centre local de services communautaires apparaît comme un ingrédient nécessaire à la réussite d'un projet de ce type. Les réactions des jeunes à cette intervention y sont aussi examinées. 
VACHON, Vivianne, intervenante sociale, Le Phare C.L.S.C. Sainte-Foy Sillery.

BERLINGUET, Marie, coordonnatrice du programme Famille/Jeunesse, Le Phare C.L.S.C. Sainte-Foy Sillery.

\section{Une intervention sur le sida dans une école secondaire ${ }^{1}$}

\section{Vivianne Vachon Marie Berlinguet}

On ne peut penser aux conséquences du sida sans se préoccuper des jeunes. Comment, en effet, les prévenir de ce mal mortel alors qu'ils sont en plein développement affectif, vivant l'immédiat avec le sentiment qu'ils sont invulnérables?

C'est à partir de cette réflexion, à l'heure où Montréal s'apprêtait à recevoir la Ve Conférence internationale sur le sida, que nous avons élaboré un projet d'une première intervention auprès des élèves de l'école secondaire de Rochebelle (Commission scolaire des Découvreurs) sise à Sainte-Foy, en banlieue de Québec.

\section{Contexte de l'intervention}

Une intervention sur le sida ne s'improvise pas. Compte tenu des tabous entourant la sexualité, des préjugés reliés à cette maladie, des peurs qu'elle provoque, il faut s'assurer d'un accord tant de la direction de l'école que des professeurs et des parents. C'est donc en premier lieu à la direction de l'école et au comité des parents que nous nous sommes adressées. Nous proposions de donner une période d'informations de 75 minutes dans les classes de IIe, IVe et Ve secondaires, le même contenu étant dispensé en III ${ }^{\mathrm{e}}$ secondaire par les professeurs. L'accueil fut positif.

Il faut dire que depuis plusieurs années, l'école de Rochebelle donne, à même le programme régulier, des contenus informatifs et éducatifs sur les relations garçons-filles, la sexualité, la contraception, 
les M.T.S. Ces thèmes sont abordés par les professeurs de sciences religieuses et morales et sont maintenant intégrés au programme de formation personnelle et sociale mis de l'avant par le ministère de l'Éducation. Notons que le sujet du sida peut également être traité par les professeurs de biologie lors de l'explication du système sanguin de même qu'à l'intérieur d'un programme de prévention des toxicomanies où il devient très approprié d'informer du mode de transmission du sida par l'utilisation de seringues contaminées.

À plusieurs reprises, les intervenantes du C.L.S.C. Sainte-Foy Sillery rattachées à l'école - infirmières et praticiennes sociales - sont invitées à animer ou à coanimer avec les professeurs les sessions portant sur des thèmes reliés à leurs compétences professionnelles respectives. L'école est partie prenante des orientations du C.L.S.C. en matière de prévention et de santé globale et c'est dans un esprit de partenariat que se discutent les ententes de services.

\section{Philosophie et objectifs de l'intervention}

Les thèmes de l'amour, de la sexualité, de la contraception, du sida ne peuvent être discutés en milieu d'éducation sans une philosophie bien établie. Dans ce cas-ci, le principe directeur est celui de la responsabilisation du jeune face à sa vie et à son comportement. Les valeurs explicitées sont le respect de la personne, sa dignité, sa capacité à faire des choix et l'acceptation des conséquences de ses actes.

La discipline du service social de même que les milieux de la santé communautaire et de l'éducation partagent cette philosophie de base. Une telle convergence facilite le travail multidisciplinaire en unifiant le sens de l'intervention.

Les objectifs de notre intervention étaient de prévenir le sida chez les adolescents et adolescentes et de développer chez eux des attitudes de compréhension et de respect envers les personnes atteintes.

On postulait, en effet, à partir de la connaissance du milieu, que plusieurs jeunes du secondaire étaient actifs sexuellement ou tout près de l'être et que, conséquemment, il fallait informer directement les étudiants des modes de transmission de la maladie. Par ailleurs, les réactions très vives de rejet et de panique survenues en d'autres milieux vis-à-vis un élève ou un professeur atteint du sida, nous ont amenées à vouloir prévenir de telles situations. Nous avons voulu faire réfléchir les jeunes sur leurs propres réactions en leur présentant le cas hypothétique d'un compagnon de classe ou d'un professeur porteur du virus. Les objectifs de notre intervention sont en lien direct avec la mission 
préventive du C.L.S.C. tant du point de vue de la santé (objectif I) que du point de vue social (objectif II).

\section{Cadre de référence et choix méthodologiques}

Pour bien situer la problématique du sida, nous avons utilisé trois documents de référence : le rapport de la Société royale du Canada ${ }^{2}$, celui du groupe de travail du ministère de la Santé et des Services sociaux ${ }^{3}$, et plus spécifiquement, le document produit par le souscomité qui a étudié les aspects psychosociaux du sida. ${ }^{4}$

Au plan théorique, nous avons utilisé les connaissances reliées à l'adolescence et, au plan méthodologique, les stratégies de l'animation et du service social.

Quant au contenu de l'intervention, une question se posait : quel est le niveau d'information des jeunes sur le sida dans un contexte d'omniprésence des mass media? Un premier sondage fait en 1988 auprès des élèves de $\|^{\mathbb{e}}$ secondaire avait démontré un intérêt certain pour ce thème et un désir d'être renseigné. Sachant que si l'on veut influencer les attitudes et les comportements, il ne suffit pas d'informer, mais aussi de faire réfléchir, nous avons donc opté pour un modèle favorisant à la fois l'information et la réflexion. En nous inspirant du principe bien connu en service social et en pédagogie qui propose de "suivre le rythme de croissance de la personne", nous avons demandé aux jeunes de répondre à six questions inscrites au tableau. Plutôt que de donner un cours magistral, nous préférions partir des réponses des étudiants et ainsi, ajouter à ce qu'ils savaient déjà, corriger de fausses notions si nécessaire et faire avancer la réflexion.

Voici les six questions qui ont servi de déclencheurs et qui, de fait, correspondent aux thèmes sur lesquels nous souhaitions l'acquisition d'apprentissages :

- comment se transmet le sida?

— peut-on attraper le sida à l'école?

- comment réagirais-tu si quelqu'un dans ta classe avait le sida ?

- comment réagirais-tu si tu apprenais que tu as le sida?

- que peuvent vivre les gens entourant les personnes qui ont le sida?

- comment pourrais-je aider un(e) ami(e) qui a le sida ?

Dans chaque classe, les élèves étaient divisés en trois groupes, l'enseignant, l'infirmière et la praticienne sociale s'étant partagé l'animation. Chaque groupe se choisissait un secrétaire qui faisait rapport en plénière. La technique de la plénière a été retenue pour permettre aux 
intervenantes de compléter l'information là où elle n'aurait pas été suffisante en petit groupe et pour aider la classe à dégager des attitudes positives communes. La projection du vidéo Je fais le point sur le sida ${ }^{5}$ terminait le cours en officialisant d'une certaine façon l'information déjà donnée et en favorisant la mémorisation par la répétition.

Nous devons commenter ici nos choix méthodologiques. La formule du petit groupe en début de rencontre nous a paru la plus adaptée à la réalité des jeunes et à la difficulté du sujet, évitant le stress du grand groupe et favorisant l'expression personnelle. La présence de deux intervenantes du C.L.S.C. peut paraître coûteuse du point de vue administratif ; toutefois, tant pour la préparation du contenu informatif que pour sa présentation, les deux disciplines sont essentielles parce que le sida est une réalité médicale et sociale, mais surtout parce qu'infirmière et intervenante sociale sont crédibles auprès des jeunes dans leurs fonctions respectives. De par leur présence continue à l'école, elles deviennent des personnes significatives, ce qui facilite leur autorité morale. Par ailleurs, la présence du professeur est essentielle au bon déroulement de l'activité et à la poursuite, en cours d'année, de l'intégration des connaissances reçues.

\section{Évaluation de l'intervention}

Nous voulions savoir si les étudiants avaient apprécié les activités de sensibilisation et connaître leurs commentaires en vue d'une action future. À cette fin, nous leur avons soumis trois questions auxquelles ils devaient répondre par écrit :

- as-tu aimé la rencontre?

- qu'as-tu appris de nouveau?

- que voudrais-tu qu'on ajoute?

À la première question, les réponses ont été affirmatives: 155 réponses positives sur 159 questionnaires remplis en $\|^{\mathrm{e}}$ secondaire, 215 sur 221 en IVe secondaire et 223 sur 237 en Ve secondaire. Si l'on considère que les questionnaires étaient anonymes et que les jeunes de cet âge ne se gênent généralement pas pour traduire leur mécontentement, on doit conclure que le niveau de satisfaction est très élevé. Cette constatation est pour nous une incitation à poursuivre les activités de ce genre.

Notons ici quelques commentaires reçus, représentatifs de l'ensemble: "j'ai appris comment le sida se transmettait", "c'était plus facile d'en parler en petit groupe", "c'est un sujet qui nous touche de près ou nous touchera de près", "nous avons pu discuter de ce que nous ressentions". 
À la question 2, 91 étudiants de $1 / \mathrm{e}$ secondaire sur 156 disent avoir appris du nouveau sur les modes de transmission lors de la rencontre de groupe; en IVe secondaire, 113 élèves sur 221 ont la même réponse et en $V^{\text {e }}$ secondaire, 84 élèves sur 237. Certains ont appris par exemple que le sida ne se transmettait pas par les toilettes ou par les moustiques!

La dernière question permettait aux étudiants de faire des suggestions. Par exemple, un certain nombre de jeunes auraient souhaité deux périodes d'informations sur le sujet (37 en IVe secondaire et 18 en $V^{e}$ secondaire); d'autres auraient aimé entendre le témoignage d'une personne sidéenne ( 30 en IVe secondaire et 34 en Ve secondaire); quelques autres ont demandé plus d'informations sur les M.T.S. (30 en IVe secondaire et 7 en Ve secondaire).

En plus des données recueillies par questionnaire auprès des jeunes, nous avons reçu des commentaires favorables des professeurs qui coanimaient les rencontres. Si l'on s'en remet à notre propre évaluation de l'activité réalisée, en nous basant sur notre expérience et notre connaissance des jeunes et du milieu scolaire, nous pouvons dire que les étudiants ont été très attentifs au sujet présenté. Ils ont participé activement aux discussions en groupe, ont paru à l'aise et ont fait montre d'un grand sérieux dans les échanges.

Par ailleurs, au plan méthodologique, nous considérons que le choix du petit groupe était approprié; d'une part, cette formule a facilité l'expression des jeunes et, d'autre part, la synergie du groupe a contribué à des échanges de plus grande qualité compte tenu que quelques-uns des élèves étaient plus renseignés et avaient déjà réfléchi sur la question du sida.

Nous devons reconnaître cependant, comme les étudiants l'ont eux-mêmes souligné, que le programme élaboré était trop chargé pour le temps disponible. Il faut dire à notre décharge que le sujet nous paraissait difficile, qu'il était nouveau pour le milieu et que nous sentions le besoin d'avoir plus de matériel que moins. Expérimentation faite, nous laisserions plus de temps à la discussion en ateliers et n'utiliserions pas de vidéo. Mais rappelons que le climat des rencontres peut varier d'une classe à l'autre, d'une école à l'autre et que l'application d'un contenu doit se faire avec souplesse. Notons également que sur le thème de la protection contre le sida, nous aurions pu mettre un accent plus important sur l'utilisation du condom.

Quant aux réflexions suscitées par la question hypothétique "Comment réagirais-tu s'il y avait quelqu'un dans ta classe qui avait le sida? ", les étudiants, quel que soit leur niveau, ont eu dans l'ensemble les mêmes réactions, à savoir la peur d'attraper la maladie et le rejet du sidéen : "Je ferais comme d'habitude, mais je m'éloignerais "; " J'éviterais de lui toucher "; "Si c'était un professeur estimé, il serait protégé, mais 
si c'était un professeur peu aimé, il serait rejeté ». Cependant, chez les étudiants plus jeunes dont le jugement s'embarrasse peu de nuances, le comportement serait plus radical et en accord avec la gang. Leurs aînés auraient un comportement plus empreint de réflexion et d'affirmation de leurs idées personnelles, ce qui nous paraît traduire les étapes normales de l'adolescence.

Soulignons enfin que le choix d'initier la formation par les questions des jeunes a été à notre avis judicieux ; cela nous a permis d'adapter nos réponses aux besoins réellement exprimés. En ce sens, il est intéressant de constater le peu de questions sur l'homosexualité, pourtant très associée au sida au début de la maladie. C'est dire que les interrogations des jeunes ne sont pas nécessairement celles des adultes et que les interventions doivent se rapprocher le plus possible du milieu.

\section{Conclusion}

Notre intervention nous a permis de constater que si les jeunes ont encore besoin d'informations, malgré l'importante couverture faite par les mass media sur le sida, c'est surtout au niveau des attitudes qu'il faut travailler, tant pour la prévention que pour la tolérance à développer face à la personne atteinte. Il faut cependant remarquer que les jeunes reflètent généralement les forces mais aussi les tabous et les préjugés de leur milieu et que c'est toute la communauté qui doit être sensibilisée à la problématique du sida.

En ce sens, le C.L.S.C. et la Commission scolaire ont présenté à l'automne 1989 la pièce de théâtre Absence ou un inconnu dans notre bande ${ }^{6}$, jouée en première à la $V^{e}$ Conférence internationale sur le sida et qui se veut un instrument pédagogique de prévention et de réflexion pour les jeunes. Cette activité a été offerte aux directions d'écoles, professeurs, comités de parents et conseils étudiants. Nous espérons qu'un programme conjoint d'activités éducatives pourra être élaboré suite à cette présentation, programme que nous souhaiterions basé sur une approche systémique, rejoignant tant les adultes que les jeunes. C'est peut-être en rapprochant les générations que la communauté sera le plus apte à vaincre le sida. 


\section{Notes et références}

1 Les personnes suivantes travaillant à Le Phare C.L.S.C. Sainte-Foy Sillery, ont participé à l'expérimentation de cette intervention: Yvonne Bérubé et Marlyse Kirouac, infirmières, Vivianne Vachon, praticienne sociale.

2 Société royale du Canada, Le sida: l'état de la question au pays, Ottawa, Société royale du Canada, 1988.

3 Groupe de travail sur le sida, Rapport synthèse, Direction des communications, Ministère de la Santé et des Services sociaux du Québec, 1988.

4 Berlinguet, M. et al., Les aspects psychosociaux du sida: problématique et intervention, Direction générale Prévention et Services communautaires, Ministère de la Santé et des Services sociaux du Québec, 1988.

5 Ce vidéo de 25 minutes est une production de Radio-Québec, 1988. Il s'adresse aux élèves du secondaire, dans le cadre des cours Formation personnelle et sociale ( $2^{\mathrm{e}}$ cycle) et Biologie (III e secondaire). Pour commande, s'adresser à : Distribution Radio-Québec, 600 rue Fullum (5e étage), Montréal, Québec, H2K $3 \mathrm{~L} 6$.

${ }^{6}$ Cette pièce de théâtre fait l'objet de l'article qui suit. 